\title{
Doubts, Fears and Misconceptions. What is the Future of Thrombolysis in Acute Stroke?
}

\author{
Philip A. Barber, Michael D. Hill, Andrew M. Demchuk, Alastair M. Buchan
}

\begin{abstract}
Alteplase for acute ischemic stroke may be the first stroke intervention to have a significant public health impact. In February 1999, this therapy was conditionally licensed in Canada for acute ischemic stroke within three hours of symptom onset. However, considerable controversy exists regarding its safety, its wider applicability outside clinical trials, and its ultimate availability. In this article we review the thrombolytic literature, attempt to answer many of the concerns, provide new guidelines for its use, and cite the need for more information about whom we should and should not be treating with this therapy.
\end{abstract}

RÉSUMÉ: Doutes, craintes et conceptions erronées. Quel est l'avenir de la thrombolyse dans l'accident vasculaire cérébral aigu? L'alteplase en phase aiguë de l'accident vasculaire cérébral (AVC) ischémique est peutêtre la première intervention dans l'AVC qui aura un impact significatif sur la santé publique. En février 1999, ce traitement a reçu une approbation conditionnelle au Canada pour le traitement de l'AVC ischémique aigu, moins de trois heures après le début des symptômes. Cependant, la sécurité du produit, son applicabilité en dehors d'essais thérapeutiques et sa disponibilité éventuelle sont très controversées. Dans cet article, nous revoyons la littérature sur la thrombolyse, nous tentons de répondre à plusieurs préoccupations, nous fournissons de nouvelles lignes directrices pour son utilisation et nous soulignons la nécessité de mieux définir les indications et les contreindications à ce traitement.

Can. J. Neurol. Sci. 2000; 27: 283-287

Stroke is the third most common cause of death and a major cause of neurological disability worldwide. Hope that the traditional nihilistic approach to acute stroke care would change followed the publication of the positive National Institute of Neurological Diseases and Stroke (NINDS) trial ${ }^{1}$ of alteplase (recombinant tissue plasminogen activator) in acute ischemic stroke. The approval of the drug by the US Food and Drug Administration, as well as endorsement by the American Academy of Neurology and the American Heart Association ${ }^{2,3}$ further increased the interest within the medical community for this new ischemic stroke treatment. The trial suggested that the benefit was substantial with an extra 16 independent survivors per 100 patients treated.

However, the results of the NINDS trial have not been replicated by other clinical trials using alteplase. ${ }^{4,5,6}$ These results have enhanced the controversy over the use of alteplase in acute stroke. While many clinicians have enthusiastically endorsed the use of alteplase for the treatment of acute ischemic stroke, others are concerned about the risk of symptomatic intracerebral hemorrhage and of the proven efficacy of the treatment in all stroke subtypes. ${ }^{7,8}$

Recently, the drug was approved in Canada for acute ischemic stroke according to the NINDS protocol. A postmarketing surveillance study, the Canadian Activase for Stroke Effectiveness Study, mandated by the Health Protection Branch as a condition of approval, is underway. ${ }^{9}$

In this article we discuss whether the enthusiasm for the use of the treatment is justified, introduce new guidelines for the use of alteplase in Canada, and provide a way to remedy the uncertainties that exist in the use of this novel agent for acute ischemic stroke.

\section{THE CURRENT LITERATURE}

The pivotal NINDS trial ${ }^{1}$ was the first positive thrombolytic study; despite an increased incidence of symptomatic intracerebral hemorrhage $(\mathrm{ICH})$ in the treatment group $(6.4 \%$

\footnotetext{
From the Calgary Regional Stroke Program, Department of Clinical Neurosciences, University of Calgary, Alberta, Canada.

ReCEIVED JANUARY 26, 2000. ACCEPTED Infinalform May 23, 2000.

Reprint requests to: Philip A Barber, Department of Clinical Neuroscience, Stroke Research, Room 1162, 1403-29 Street NW, Calgary, Alberta, Canada T2N 2T9
} 
receiving treatment versus $0.6 \%$ in controls), treatment with intravenous alteplase within three hours of the onset of ischemic stroke significantly improved clinical outcome at three months. Longitudinal data suggest benefit out to 12 months. ${ }^{10}$

The initial study was, in fact, two trials. Part 1 tested whether alteplase had clinical activity, indicated by an improvement at 24 hours from stroke onset of four points over baseline values of the National Institute of Health Stroke Score (NIHSS) or the resolution of the neurological deficit. Part 2 used a global test statistic (combining four accepted stroke scales) to assess clinical outcome at three months.

While the results from Part 2 are straightforward with an Odd's Ratio of 1.7 in favour of treatment, in Part 1, there was no significant difference between the percentage of patients with neurological improvement at 24 hours (although benefit was observed for these patients at three months for all of the predefined end points). This neutral result in Part 1 caused some to question the treatment benefit observed at the three-month follow up. However, this dichotomy was likely due to the choice of an insensitive endpoint. The median NIHSS score improvement in both Part 1 and 2 at 24 hours were significantly better than the placebo group. The originally defined four-point threshold for declaring early neurological improvement was unduly conservative, a factor that many of the investigators explained by the absence of good natural history data available prior to the design of the trial. If the analysis is repeated and the threshold for neurological improvement is varied between four and 14 points, above a threshold of five points, the difference in proportion of patients with early neurological improvement in favour of alteplase is consistently significant. These results were replicated in Part 2. ${ }^{11}$ Therefore both placebo and alteplase treated patients showed neurological improvement at 24 hours, but alteplase treated patients improved more.

In the European Cooperative Acute Stroke Study (ECASS) I, ${ }^{4}$ patients were enrolled within six hours of stroke in a randomised, double blind, placebo-controlled trial. Eighty percent were treated more than three hours after stroke. Those assigned to active treatment received alteplase in dose of $1.1 \mathrm{mg} / \mathrm{kg}$ up to a total of $100 \mathrm{mg}$. The study failed to find a significant benefit in the intention to treat analysis for the primary end points (Barthel Index and modified Rankin Scale scores). However, a proportion of patients actually violated the inclusion criteria as determined by the presence of early signs of infarction on the CTscan. When these patients were excluded, benefit was seen for the treated patients (target population).

In 1998, ECASS II was published. ${ }^{5}$ This trial enrolled 800 patients in Europe, Australia and New Zealand, and randomised them to alteplase $0.9 \mathrm{mg} / \mathrm{kg}$ or placebo within six hours of symptom onset. The investigators were successful in excluding patients with early infarct signs (as defined from ECASS I). However, in this study, $80 \%$ of patients were enrolled late, between three to six hours of symptom onset. No significant treatment benefit was seen in the primary end point. In a post hoc analysis using independent recovery, (Rankin 0-2), a significant benefit was seen with $54 \%$ of the alteplase-treated patients versus $46 \%$ placebo being independent.

The Alteplase Thrombolysis for Acute Non-interventional Therapy for Acute Ischemic Stroke Part B (ATLANTIS) ${ }^{6}$ is the third randomised trial evaluating alteplase with the majority of patients being treated between three to five hours. A comparison of the ATLANTIS Part B trial with ECASS II trial suggests that the two trial populations were quite similar with baseline median NIHSS scores of 10 and 11 respectively. The major reason that both ECASS II and ATLANTIS were unable to show a treatment effect was that the incidence of spontaneous recovery in the placebo group was $40 \%$ in both studies probably because relatively mild strokes were recruited. However, in the ATLANTIS trial, ${ }^{6}$ treatment with alteplase produced a significant increase in the percentage of patients in the target population with major neurological improvement (defined as an 11-point improvement or full recovery).

The major concern regarding the use of alteplase is the balance of risk and benefit. In all four alteplase acute stroke trials, allocation to alteplase was associated with an excess of early fatal intracranial hemorrhages ( $1.3 \%$ in controls vs. $4.4 \%$ in treated patients) representing an excess of 31 per 1000 patients treated. ${ }^{11}$ Available data on symptomatic intracranial hemorrhage in the first 36 hours, in all time windows, shows an increase from $3 \%$ in controls to $10 \%$ in treated patients, representing an excess of 73 (95\% CI 55 to 90) bleeds per 1000 treated. While treatment with alteplase leads to early excess $\mathrm{ICH}$, this is counterbalanced by a lower frequency of postischemic space-occupying oedema. ${ }^{5}$ This may reflect hemorrhagic transformation of large infarcts with severe brain oedema in patients treated with alteplase. Nevertheless, the overall benefits in these studies were substantial, with $58 \%$ dead and dependent in the control group compared to $51 \%$ in the alteplase group, representing an extra 76 (95\% CI 38 to 113) independent survivors per 1000 treated out to six hours. ${ }^{12}$ The benefit takes into account the increase in symptomatic intracerebral hemorrhage.

In ECASS II, NINDS, and ATLANTIS trials, there was no increase in either early or late mortality. It may, therefore, be argued that the greater risk to the patient may come from withholding alteplase treatment. But can alteplase be justified in every stroke patient? It seems unlikely that every stroke patient has a three-fold increase risk of symptomatic intracranial bleeding. For some patients the risk is much less or even negligible, for others it is much higher. While it is becoming clear that greater early CT ischemic change implies a higher risk of symptomatic intracerebral hemorrhage, ${ }^{13,14}$ further trials and experience are needed to identify specific patient subgroups that are more or less likely to benefit.

\section{Feasibility}

Despite the proven efficacy in the NINDS trial, use of this therapy remains limited by concerns about its safety outside a carefully controlled clinical study. A study of over 1000 patients treated in 30 stroke centres demonstrated a $6 \%$ symptomatic ICH rate, ${ }^{15}$ a rate similar to that observed in the NINDS trial. These figures, however, vary among studies. Preliminary analysis, for instance, from the Minnesota community project identified symptomatic ICH in $9 \%$ of patients with $6 \%$ being fatal. ${ }^{16}$

As with other treatments such as endarterectomy for symptomatic carotid stenosis, and anticoagulation for atrial fibrillation, the benefits are dependent on the complication rate being low. ${ }^{17,18}$ Similarly, with thrombolysis for acute ischemic stroke, the benefit is related to patient selection, physician expertise and the adherence to the NINDS protocol. ${ }^{19}$ The 
potential public health impact or cost effectiveness of alteplase in acute ischemic stroke is not known.

In the NINDS trial, only $4 \%$ of those screened were eligible for the study. Many have argued that because of the limited time window and restrictions of eligibility, treatment with alteplase may only benefit single patients but will have no impact on the general prognosis of stroke. ${ }^{20} \mathrm{~A}$ similar situation arose with the use of thrombolytic therapy in acute myocardial infarction (MI) in the early 1980s. Even after there was convincing evidence regarding the efficacy of thrombolysis in MI only approximately $10 \%$ of eligible patients were being treated in North America. ${ }^{21}$

Enthusiasm and confidence in the treatment are required to provide an infrastructure that allows greater patient access to the therapy. Recently, the NINDS group presented evidence that earlier treatment is better. ${ }^{22}$ In Cologne, ${ }^{23}$ a referral system was developed which directed eligible patients throughout the city to a highly experienced acute stroke service. In this setting, more than $20 \%$ of referred patients were treated with alteplase within three hours with one in four treated within 90 minutes of symptom onset.

\section{NEWTHROMBOLYSIS GUIDELINES}

As clinicians become more familiar with the use of this therapy in Canada and more information becomes available about the use of alteplase in acute stroke, there is a need to continuously revise the initial recommendations for the use of alteplase. ${ }^{24}$ For instance, it is no longer a requirement to have neurosurgical facilities available on a 24 hour basis to manage hemorrhagic complications. This was never a stipulation of the NINDS protocol, it has practical difficulties and experience has suggested that neurosurgical intervention is rarely undertaken in the setting of symptomatic $\mathrm{ICH}^{25}$ Similarly, neuroradiological expertise is not required on a 24 hour basis to aid in the detection of early CT ischemia and $\mathrm{ICH}$ as long as the attending clinician is both comfortable and familiar with the radiological features. We feel it necessary to remove the CT exclusion of ischemia involving greater than $33 \%$ middle cerebral artery territory because of its poor reliability. ${ }^{13,26-29}$ We propose the use of the Alberta Stroke Program Early Computed Tomography Score to quantify early CT ischemic change which has better interobserver reliability compared to the $1 / 3$ middle carotid artery territory. ${ }^{14}$ Treatment can be justified in the presence of early CT ischemic change but the risk of symptomatic ICH increases. The only broad recommendation that should remain is for the therapy to be administered by a physician with expertise in the diagnosis and management of acute stroke. Our current suggested guidelines are summarised in the Table.

\section{THe FUTURe}

While we can recommend the use of alteplase for acute ischemic stroke patients within three hours of symptom onset in centres that have proven expertise and experience, there remain considerable uncertainties. Intravenous alteplase is not a panacea for all stroke subtypes. It is important to identify clinical criteria that can help identify those at high risk of intracerebral hemorrhage. Uncertainties also exist regarding other factors, for instance, the very elderly, stroke severity, blood pressure management and the therapeutic tissue window. Clearly, some patients that were treated between three to six hours did benefit as indicated by the post hoc analysis of ECASS II, but can they be identified?

Developments are also taking place in the field of neurovascular imaging, which has paralleled renewed interest in the concept of the ischemic penumbra. A promising noninvasive approach that combines high-resolution imaging with the possibility of repeated measurements is diffusion weighted imaging. ${ }^{30}$ However, while MRI will offer important insights into the pathophysiology of acute stroke, its current practical use is limited by restricted accessibility and there is no evidence that

Table: Practical guidelines for the use of alteplase for acute ischemic stroke

\section{Inclusion criteria:}

- Acute ischemic stroke with clearly definable time of onset

- Patient presenting early enough so that thrombolytic infusion may be started within three hours from symptom onset

\section{Absolute contraindications:}

- Pre-treatment CT scan on 3rd or 4th generation scanner showing: hemorrhage, mass effect or oedema, tumour, or arteriovenous malformation

- TIAor stroke with rapidly improving deficit

- History and examination compatible with subarachnoid hemorrhage

- Blood pressure > 185/110 after two attempts to reduce BP to this level or below

- Major surgery or trauma in the last 14 days

- Previous history of intra-parenchymal CNS bleeding

- Intracranial surgery or intraspinal surgery < two months

- Active internal bleeding

- Arterial puncture at a noncompressible site in the last seven days

- History of haematological abnormality OR coagulopathy, OR anticoagulation for any reason $(\mathrm{PT}>15 \mathrm{sec}$, INR $>1.7$, PTT $>40 \mathrm{sec}$, platelets $<100 \times 10^{\wedge} 9 / 1$ )

- Previous anaphylactic response to alteplase

Warnings (i.e. consider risks and benefits):

- The rare instance of the CT scan showing obvious hypodensity involving most of the MCAterritory (low ASPECTS)

- Stroke or head injury (skull fracture or concussion) in the preceding three months

- Recent transmural myocardial infarction in the previous two months (increased risk of cardiac tamponade)

- History of GI or GU hemorrhage in the prior 21 days

- Glucose $<2.7 \mathrm{mmol} / \mathrm{l}$ or $>22.2 \mathrm{mmol} / \mathrm{L}$ as extremes of glycaemia can mimic stroke symptoms

- Seizure at stroke onset because this may mimic stroke symptoms or confuse the severity of the stroke

- Pregnancy

- Endocarditis (risk of bleeding into septic foci), acute pericarditis (risk of cardiac tamponade)

- Serious underlying medical illness which may make the administration of alteplase dangerous (liver failure), or which may restrict the potential benefit (co-morbid factors). 
it will allow safer administration of thrombolytics. What may improve acute stroke care is information regarding the vascular pathology which will allow the monitoring of recanalisation induced by thrombolysis.

Transcranial doppler, CT angiography and MR angiography can diagnose vascular occlusions safely, quickly, accurately and specifically. ${ }^{31}$ The detection and monitoring of a vascular occlusion is of particular relevance following the publication of the positive Prolyse in Acute Thromboembolism II trial which used intra-arterial prourokinase and has demonstrated that the therapeutic window for a significant number of patients may extend to six hours in highly selected stroke patients. ${ }^{32}$

Currently, from a world perspective, the attitudes towards alteplase treatment for acute ischemic stroke within Canada lie somewhere between the overwhelming enthusiasm for the treatment as seen in the US and the entrenched nihilism seen in parts of Europe. ${ }^{33}$ If the NINDS trial, by the play of chance, overestimated the benefits of alteplase, the slow uptake of thrombolysis around the world may be an appropriate response. Similarly, if the selection criteria that are now being applied to the use of alteplase are unduly restrictive, then a large number of patients will be denied an effective therapy. Some investigators have suggested that many of these insecurities can be potentially answered by the International Stroke Trial III which proposes to randomise 6000 patients in a placebo-controlled study within six hours of stroke symptom onset. The proposed trial is in danger of being a further three to six hour study because of the ethical concerns of randomising patients presenting within three hours of symptom onset in countries where the therapy has received approval, such as the US and Canada. There are doubts that a large study will help us understand why some patients do not respond to alteplase. Future stroke trials should concentrate on improved patient selection based on our current understanding of the pathophysiology of the disorder through the use of noninvasive neuroimaging.

\section{REFERENCES}

1. National Institute of Neurological Disorders and Stroke rt-PA Stroke Study Group. Tissue plasminogen activator for acute ischemic stroke. N Engl J Med 1995; 333:1581-1587.

2. Adams HP, Brott TG, Furlan et al. Guidelines for thrombolytic therapy for acute ischemic stroke: a supplement to the guidelines for the management of patients with acute ischemic stroke. Stroke 1996; 27: 1711-1718.

3. Report of the Quality Standards Subcommittee of the American Academy of Neurology. Practice advisory: thrombolytic therapy for acute ischemic stroke - Summary Statement. Neurology 1996; 47: 835-839.

4. Hacke W, Kaste M, Fieschi C, et al, for the ECASS Study Group. Intravenous thrombolysis with recombinant tissue plasminogen activator for acute hemispheric stroke: The European Cooperative Acute Stroke Study (ECASS). JAMA 1995; 274:1017-1025

5. Hacke W, Kaste M, Fieschi C, et al. Randomised double blind placebo-controlled trial of thrombolytic therapy with intravenous alteplase in acute ischaemic stroke (ECASS II). Lancet 1998; 352: $1245-1251$.

6. Clark WM, Wissman S, Albers GW, et al. Recombinant tissue-type plasminogen activator for ischemic stroke 3-5 hours after symptom onset. JAMA1999;282:2019-2026.

7. Caplan LR, Mohr JP, Kistler JP, Koroshetz, Grotta J. Clinical debate: should thrombolytic therapy be the first-line treatment for acute ischemic stroke? N Engl J Med 1997;337: 1309-1313.
8. Devuyst D, Bogouslavsky J. Recent progress in drug treatment for acute stroke. J Neurol Neurosurg Psychiatry 1999; 67 (4): 420-425.

9. Hill MD, Barber PA, Buchan AM for the CASES Investigators. The Canadian Activase for Stroke Effectiveness Study (abstract). Stroke 2000: 31: 315

10. Kwiatkowski TG, Libman RB, Frankel M, et al. Effects of tissue plasminogen activator for acute ischemic stroke at one year. $\mathrm{N}$ Engl J Med 1999; 340:1781-1787.

11. Haley Jr, Lrwnadowski C, Tilley BC. NINDS rt-PA Stroke Study Group. Myths regarding the NINDS rt-PA Stroke Trial: Setting the record straight. Ann Emerg Med 1997;30:676-682.

12. Wardlaw JM, Yamaguchi T, del Zoppo G. Thrombolytic therapy versus control in acute ischaemic stroke. Stroke module of the Cochrane Database of Systematic Reviews. Available in the Cochrane Library (database on disk and CDROM). Oxford: Update software, 1999.

13. von Kummer R, Allen KL, Holle R, et al. Acute stroke: usefulness of early CT findings before thrombolytic therapy. Radiology 1997; 205: 327-333

14. Barber PA, Demchuk AM, Zhang J, Buchan AM on behalf of the ASPECTS study group. The validity and reliability of a quantitative CTscore predicts outcome in hyperacute stroke prior to thrombolytic therapy. Lancet 2000;355:1670-1674.

15. Tanne D, Demchuk A, Kasner S, Grond M, et al. A large multinational investigation to predict t-PA related symptomatic ICH in patients with acute ischemic stroke (abstract). Stroke 1999;30:248.

16. Hanson SK, Brauer DJ, Anderson DC, et al. Stroke treatment in the community (STIC) - Intravenous rt-PA therapy for acute stroke in clinical practice. Neurology 1998; 50:A155 (abstract).

17. Barnett HJ, Taylor DW, Eliasziw, et al. Benefit of carotid endarterectomy in patients with symptomatic or severe stenosis. North American Symptomatic Carotid Endarterectomy Collaborators. N Engl J Med 1998;339:1415-1425.

18. Risk factors for stroke and efficacy of antithrombotic therapy in atrial fibrillation. Analysis of pooled data from five randomised controlled trials. Arch Intern Med 1994;154:1449-57.

19. Buchan AM, Barber PA, Karbalai HG, et al. Effectiveness of t-PA in acute ischemic stroke: outcome relates to appropriateness. Neurology 2000; 54:679-684.

20. Jorgenson HS, Nakayama H, Kammersgaard LP, Raaschou HO, Olsen TS. Predicted impact of intravenous thrombolysis on prognosis of general population of stroke patients: simulation model. Br Med J 1999; 919: 288-289.

21. Doorey AJ, Michelson EL, Topol EJ. Thrombolytic therapy of acute myocardial infarction. JAMA1992;268(21):3108-3114.

22. Marler J, Tilley B, Mei L, et al. Earlier treatment associated with better outcome in the NINDS TPA stroke study (abstract). Stroke 1999; 30:244

23. Grond M, Rudolf J, Schmulling S, et al. Can the NINDS results be transferred into daily routine? Stroke 1998;29:288 (abstract).

24. Norris J, Buchan AM, Cote R, et al. Canadian guidelines for intravenous thrombolytic treatment with acute stroke. Can J Neurol Sci 1998; 25: 257-259.

25. Hill MD, Barber PA, Demchuk AM, et al. The Calgary Regional Stroke Program: Building a brain attack team for thrombolytic therapy in acute ischaemic stroke. Can Med Assoc J 2000;162(11):1589-1593.

26. Dippel DWJ, Du RY, van Beest Holle M, et al. The validity and reliability of early infarct signs on CT in acute ischaemic stroke. Cerebrovas Dis 1997;7 (suppl 4):15.

27. Schriger D, Kalafut M, Starkman S, et al. Cranial computed tomography interpretation in acute stroke. Physicians accuracy in determining eligibility for thrombolytic therapy. JAMA 1998 ; 279:1293-1297.

28. Grotta J, Chiu D, Lu M, Patel S, et al. Agreement and variabilty in the interpretation of early CT changes in stroke patients qualifying for intravenous rt-PA. Stroke 1999;30:1528-1533.

29. Wardlaw JM, Dorman PJ, Lewis SC, Sandercock PAG. Can stroke physicians and neurologists identify signs of early cerebral infarction on CT? J Neurol Neurosurg Psychiatry 1999; 67:651653. 
30. Beaulieu C, de Crespigny A, Tong DC, et al. Longitudinual magnetic resonance imaging study of perfusion and diffusion in stroke: evolution of lesion volume and correlation with clinical outcome. Ann Neurology. 1999;46:568-578.

31. Yamaguchi T, Mori E, Minematsu K, del Zoppo, eds. Thrombolytic Therapy in Acute Ischemic Stroke III. Tokyo: Springer-Verlag, 1995.
32. Furlan A, Higashida R, Wechsler L, et al. Intra-arterial prourokinase for acute ischemic stroke. The PROACT II study: a randomised controlled trial. JAMA1999; 282: 2003-2011.

33. Anonymous. Royal College of Physicians of Edinburgh consensus conference on medical management of stroke, 26 and 27 May 1998. J Neurol Neurosurg Psychiatry 1999; 66:128-129. 